\title{
SHARP REFRACTORY COMPOSITE LEADING EDGES ON HYPERSONIC VEHICLES
}

\author{
Sandra P. Walker \\ NASA Langley Research Center, Hampton, VA \\ Brian J. Sullivan \\ Materials Research and Design, Rosemont, PA
}

\begin{abstract}
$\underline{\text { ABSTRACT }}$
On-going research of advanced sharp refractory composite leading edges for use on hypersonic air-breathing vehicles is presented in this paper. Intense magnitudes of heating and of heating gradients on the leading edge lead to thermal stresses that challenge the survivability of current material systems. A fundamental understanding of the problem is needed to further design development. Methodology for furthering the technology along with the use of advanced fiber architectures to improve the thermalstructural response is explored in the current work. Thermal and structural finite element analyses are conducted for several advanced fiber architectures of interest. A tailored thermal shock parameter for sharp orthotropic leading edges is identified for evaluating composite material systems. The use of the tailored thermal shock parameter has the potential to eliminate the need for detailed thermal-structural finite element analyses for initial screening of material systems being considered for a leading edge component.
\end{abstract}

\section{INTRODUCTION}

Advanced air-breathing hypersonic vehicles require sharp leading edges to meet aerodynamic performance requirements. An example of an air-breathing hypersonic vehicle concept is illustrated in Figure 1. Circled are some locations on the vehicle requiring sharp leading edge technology. Flight trajectories having rapid acceleration to Mach 10 impose significant thermal stresses along with operating temperatures exceeding $3800^{\circ} \mathrm{F}$ on sharp leading edges. To meet advanced vehicle requirements, technology development for the leading edge structure has been identified as essential in Reference 1 .

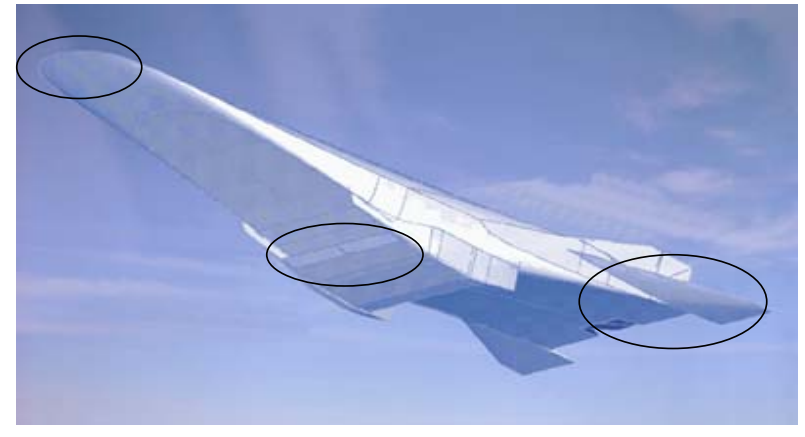

Figure 1: Air-breathing hypersonic vehicle concept.

Ceramic matrix composites utilizing carbon fibers are candidate material systems for sharp leading edge applications under hypersonic flight conditions. Although the use of various ceramic matrix materials requires investigation for identification of an optimum material system, the focus of this preliminary effort is limited to carbon matrix composites utilizing carbon fibers $(\mathrm{C} / \mathrm{C})$.

The application as a nose leading edge for an air-breathing vehicle demonstrator is used as a basis for this study. Development of the $\mathrm{C} / \mathrm{C}$ material system is pursued through investigating the effect of hybrid fiber architectures on improving the thermal-structural response for a sharp leading edge. A theoretical, semi-empirical micro-mechanics approach is utilized to predict the material properties of the advanced $\mathrm{C} / \mathrm{C}$ material system.

Detailed three-dimensional thermal and structural finite element analyses are conducted on the sharp leading edge component to predict the critical thermal stresses and thermal buckling loads. Factors of safety are determined through comparison of predicted thermal stresses with material strength allowables. 
An effort to identify desirable material attributes for improved factors of safety led to the identification of a thermal shock parameter for orthotropic sharp leading edges. The thermal shock parameter is evaluated here as a material screening tool for sharp leading edges.

The test specimen requirements to structurally validate sharp leading edges are considered. Initial material property testing requirements are defined for screening materials. Additionally, leading edge specimen width requirements for replicating critical thermal stress magnitudes are identified. The fabrication of advanced hybrid $\mathrm{C} / \mathrm{C}$ test panels is described. The material specifications are presented and two architectures of interest were chosen for fabrication.

\section{AERODYNAMIC HEATING ENVIRONMENT}

The aerodynamic heating conditions from a specific Mach 10 flight trajectory are utilized in this study. The leading edge under evaluation has a tip radius of 0.03 inches, is unswept and operating at zero angle of attack. Figure 2 shows the stagnation point heat flux on the tip of the sharp leading edge. In addition to the magnitude of the peak heat flux being intense, the temporal gradient of the heat flux is also intense. Consequently, the leading edge will also be considered here as being subject to a thermal shock condition.

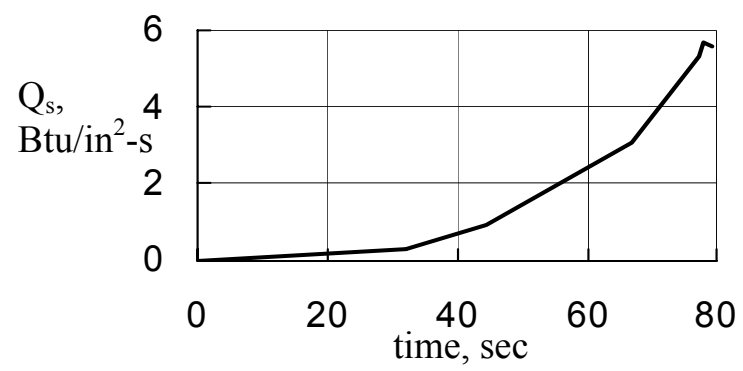

Figure 2. Stagnation heat flux for a Mach 10 flight trajectory.

\section{BASELINE FINITE ELEMENT ANALYSIS}

Three-dimensional finite element thermal and structural analyses were conducted to predict the response of material systems of interest. The geometry of the leading edge being analyzed is illustrated in Figure 3. The leading edge component is four inches long in the chordwise, $\mathrm{x}$ direction (material 1-direction), 18 inches wide in the spanwise, y-direction (material 2-direction), and 0.5 inches thick in the z-direction (material 3direction) at the root. The tip of the leading edge has a radius on 0.03 inches. Due to symmetry in the spanwise direction, only one-half of the leading edge width was modeled. The leading edge attachment region was 0.251 inches thick in the $\mathrm{z}$-direction and 0.864 inches long in the $\mathrm{x}$ direction. A commercially available finite element analysis code, NASTRAN, was utilized for the analyses. $^{2}$ The finite element model contained a total of 20,880 eight-node hexahedral solid elements.

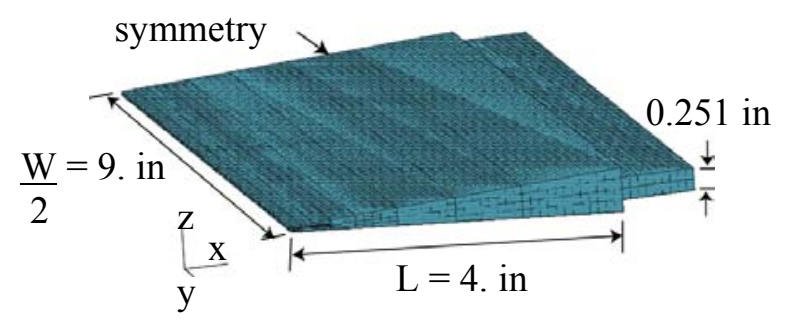

Figure 3. Leading edge analysis model.

A baseline $\mathrm{C} / \mathrm{C}$ material system with a $3: 1$ ratio of pitch type fibers was first analyzed. The $3: 1$ ratio identifies the ratio of chordwise to spanwise fibers in a ply weave. All pitch (K321) fibers were utilized in the $3: 1$ weave. The pitch fibers were chosen for their high thermal conductivity in comparison to other commercially available fibers, and thus were utilized to aid in reducing the peak temperatures occurring at the stagnation point on the tip of the leading edge. Accordingly, the material was oriented with the higher density of fibers chordwise (i.e., $\mathrm{x}$ direction) .

\section{Thermal Analysis}

A transient thermal analysis was conducted on the leading edge model assuming an initial temperature of $0^{\circ} \mathrm{F}$. The temperature dependent thermal conductivity of the material was modeled. The heat flux consisted of a predicted chordwise distribution on the upper and lower surfaces using the transient stagnation heat 
flux (Figure 2) at the tip and applied uniformly across the span of the leading edge. The surfaces at $y=0$ and $y=9$. inches, the end surface at $x=L$ and the attachment region were assumed adiabatic. Radiation to space was modeled with a surface emissivity of 0.75 .

Since a uniform heat flux was applied in the span direction, there was no spanwise temperature gradient predicted and the predicted temperature gradient occurring through the thickness was also insignificant. The predicted temperature distribution in the $\mathrm{x}$-direction at the time, $\mathrm{t}=79 \mathrm{sec}$ is displayed in Figure 4. A peak temperature of $\mathrm{T}=3800^{\circ} \mathrm{F}$ occurred at the time, $\mathrm{t}$ $=79 \mathrm{sec}$. As can be seen in Figure 4, a severe temperature gradient occurs in the chordwise direction at the tip of the leading edge.

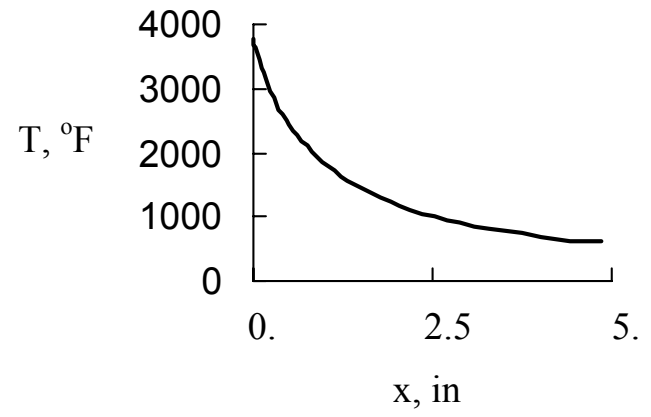

Figure 4. Temperature distribution at $\mathrm{t}=79 \mathrm{sec}$.

\section{$\underline{\text { Structural Analysis }}$}

Non-linear static structural analyses were conducted for the leading edge using temperature dependent material properties. The attachment area was allowed to freely expand and only free body motion was constrained in the analysis. The unconstrained boundary condition was expected to closely replicate the actual attachment configuration constraint. The critical thermal stresses were predicted to occur at the same time, $t$ $=79 \mathrm{sec}$, as the peak temperatures occurred. The critical maximum stress was compressive occurring in the spanwise direction at the center on the tip of the leading edge. A contour plot of the critical $\sigma_{\mathrm{y}}$ stress distribution is displayed in Figure 5. The predicted peak $\sigma_{\mathrm{ymax}}$ was significantly greater than the material allowable with a factor of safety of 0.42 , implying the design was expected to fail.
A linear bifurication buckling analysis was also performed for these loading conditions. The results revealed no concern with thermal buckling of the leading edge design.

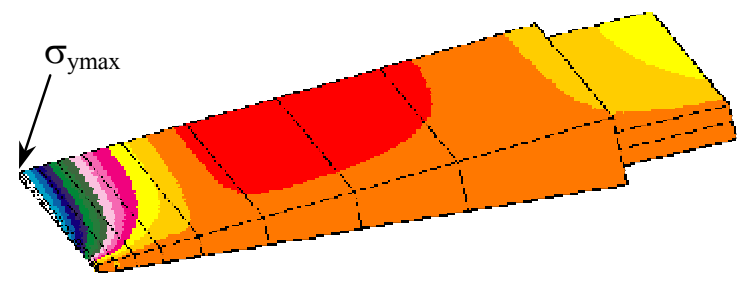

Figure 5. Spanwise stress distribution at $t=79$ sec.

\section{ALTERNATE FIBER ARCHITECTURE EVALUATION}

Because the baseline composite
architecture was predicted to fail due to
compressive thermal stresses, advanced fiber
architectures for improved thermal and structural
response were investigated.

Fiber Selection Considerations

The key issues related to the thermal and structural response of the composite sharp leading edge include: 1) peak temperature, and 2) peak compressive $\sigma_{y}$, both occurring at the tip of the leading edge.

The desire to limit the peak temperature is driven by both the operational limit of the coatings to resist oxidation and the effect of excessive temperature degrading structural performance. Consequently, the fiber architecture should optimally employ high conductivity pitch fibers chordwise to transfer heat away from the tip and reduce peak temperatures for improved material behavior. The ratio of fibers in the chordwise direction to fibers in the spanwise direction should also be the maximum possible with permissible factors of safety for the design. The use of the high conductivity fiber additionally benefits the design yielding lower spatial temperature gradients and hence thermal stresses at the tip.

An increase in the spanwise compressive allowable stress will also improve the critical factor of safety for the leading edge design. Consequently, employing T-300 fibers spanwise would be desirable since the T-300 fibers 
generally have a higher compressive strength while at the same time possessing a lower compressive modulus then other available fibers. This remains the case even when the T-300 fibers are heat-treated, as would be necessary for their use in this high temperature application. Note that as-received T-300 fibers are processed at much lower temperatures than they will experience in this composite application.

Based on the aforementioned issues relevant to sharp leading edges, hybrid fiber architectures, utilizing pitch fibers chordwise, weaved with T-300 fibers spanwise, are being evaluated here for improving the thermalstructural response of the leading edge. In addition to using T-300 fibers spanwise, the use of varying fiber orientation requires investigation. Although optimal ply angle orientation requires future consideration, the current investigation is limited to incorporating $\pm 45^{\circ}$ plies of T-300 fibers in a 1:1 weave in the fiber architecture design.

\section{Material Property Predictions}

Material properties predictions are needed for the composite $\mathrm{C} / \mathrm{C}$ material systems to conduct the three-dimensional thermal and structural finite element analyses. For each fiber architecture to be evaluated, the predicted temperature-dependent properties include: 1) thermal conductivity, $\mathrm{k}_{\mathrm{x}}, \mathrm{k}_{\mathrm{y}}$, and $\mathrm{k}_{\mathrm{z}}, 2$ ) engineering modulus, $\mathrm{E}_{\mathrm{x}}, \mathrm{E}_{\mathrm{y}}, \mathrm{E}_{\mathrm{z}}, 3$ ) Poisson's ratios, $\left.v_{\mathrm{yx}}, v_{\mathrm{zx}}, v_{\mathrm{zy}}, v_{\mathrm{xy}}, v_{\mathrm{xz}}, v_{\mathrm{yz}}, 4\right)$ shear modulus, $\left.G_{x y}, G_{x z}, G_{y z}, 5\right)$ coefficient of thermal expansion, $\left.\alpha_{x}, \alpha_{y}, \alpha_{z}, 6\right)$ tensile strength $F_{t x}, F_{t y}$, $\left.\mathrm{F}_{\mathrm{tz}}, 7\right)$ compressive strength, $\mathrm{F}_{\mathrm{cx}}, \mathrm{F}_{\mathrm{cy}}, \mathrm{F}_{\mathrm{cz}}$, and 8) shear strength, $T_{x y}, T_{x z}, T_{y z}$, where subscripts $x, y$, and $\mathrm{z}$ are the three material coordinate directions.

The method for predicting the material properties utilizes a database containing test data from numerous $\mathrm{C} / \mathrm{C}$ composites. Test data included data for $\mathrm{C} / \mathrm{C}$ composites that employ the pitch (K321 or P30X) type fibers ${ }^{3}$, and separately, data on $\mathrm{C} / \mathrm{C}$ composites that employ the T-300 fibers ${ }^{4}$. The data was used to back-calculate the effective fiber and matrix properties. Using classical micromechanical methods ${ }^{5,6}$, the fiber and matrix properties were then projected to predict thermo-elastic and thermal conductivity properties for the hybrid two-dimensional laminate constructions of interest. Composite strength predictions were also performed using the backcalculated properties in the candidate hybrid composites. This involves the analysis of unit cell models of the hybrids under multiple unidirectional stress states to calculate the stresses necessary to cause failure of the composite for each stress component. ${ }^{7}$ In these calculations, particular emphasis was placed on the material characterization in the vicinity of $4000^{\circ} \mathrm{F}$.

\section{Evaluation Procedure}

The procedure for evaluating the refractory composite material systems under consideration include the following steps:

1. Identify laminate construction for evaluation

2. Generate material properties for analyses

3. Conduct transient thermal analysis of leading edge subject to aerodynamic heating environment

4. Conduct structural analyses to predict critical thermal stresses

5. Compute factors of safety for material system

6. Repeat 1-5 for each material system being evaluated

7. Compare factor of safety and peak temperature for each material system to identify best material for application of interest

\section{THERMAL SHOCK PARAMETER}

The attempt has been made to define a material parameter as a screening tool for evaluating fiber architecture designs. Use of a material parameter to rank the designs could possibly eliminate the need for detailed thermal and structural analyses of every architecture being evaluated and thus improve design efficiency. The material parameter could also provide insight on achieving material systems for optimal thermalstructural response of the sharp leading edge.

Considering first the material properties significantly affecting the critical factor of safety,

$$
\mathrm{FS}_{\text {critical }}=\frac{\mathrm{F}_{\mathrm{cy}}}{\sigma_{\mathrm{y} \max }}
$$

The desire to increase the critical FS would be to maximize the right hand side of Equation (1). The peak $\sigma_{\text {ymax }}$ can be roughly approximated to be 
dominated by the term $\mathrm{E}_{\mathrm{y}} \alpha_{\mathrm{y}} \Delta \mathrm{T}$, where $\Delta \mathrm{T}$ is the change in temperature from the initial temperature. This rough approximation can be justified by reasoning that the tip of the leading edge is being constrained from spanwise expansion by the cooler mass aft of the tip, which is at both lower temperatures and has a lower coefficient of thermal expansion. Finally, $\Delta \mathrm{T}$ is most sensitive to $\mathrm{k}_{\mathrm{x}}$, the thermal conductivity in the chordwise direction. This property is very dependent on fiber architecture and is inversely related to $\Delta \mathrm{T}$. Substituting the material property relations in the factor of safety ratio yields the material parameter,

$$
\mathrm{TSP}=\frac{\mathrm{F}_{\mathrm{cy}} \mathrm{k}_{\mathrm{x}}}{\mathrm{E}_{\mathrm{y}} \alpha_{\mathrm{y}}}
$$

The parameter is denoted as "TSP" because the parameter has the same material properties as the traditional thermal shock parameter identified for isotropic flat plates subject to thermal shock conditions. ${ }^{8}$ However, care should be taken in interpreting this parameter as a thermal shock parameter. In Reference 9, it was shown that a general thermal shock parameter for composite laminated plates cannot be explicitly expressed. Also, the thermal shock problem has an instantaneous step change in heating conditions on a relatively thin flat plate. Although the change in heat flux is not instantaneous here, the steep temporal gradient (Figure 2) should be further justification of considering thermal shock as an issue and utilizing a tailored thermal shock parameter for evaluating sharp leading edge materials. Consequently, the merit of a tailored thermal shock parameter (TSP) for orthotropic leading edges requires investigation for use of the parameter in screening material systems specifically for sharp leading edges and is evaluated subsequently.

\section{FIBER ARCHITECTURE THEORETICAL EVALUATION RESULTS}

Several fiber architectures were identified for evaluation based on the considerations previously presented. For each architecture, the evaluation procedure outlined previously was followed and the tailored thermal shock parameter was computed. However, complicating matters are the temperature dependent nature of the material properties of the material systems. Although the appropriate temperatures to use may require future evaluation, for this preliminary investigation, the mechanical properties $\left(F_{c y}, E_{y}\right.$, and $\left.\alpha_{y}\right)$ of the material at the baseline peak temperature of $3800^{\circ} \mathrm{F}$ were used to compute the tailored thermal shock parameter. Alternatively, since the peak temperature rise is to a greater extent affected by the thermal conductivity, $\mathrm{k}_{\mathrm{x}}$, over the transient temperature history, the initial decision here was to use the value of $\mathrm{k}_{\mathrm{x}}$ predicted at $\mathrm{T}=3530^{\circ} \mathrm{F}$, in the TSP computation, since that value is closer to an average conductivity.

Results of the fiber architecture evaluations are presented in Table 1 . The first column in the table gives the weave ratio of spanwise K321 type to chordwise T-300 type fibers. The first hybrid listed shown with a 1:0 weave were actually unidirectional layers of K321 fibers. The second column gives the percentage of fibers contained in the laminate oriented at \pm 45 degrees. The \pm 45 degree fibers were within layers weaved with a $1: 1$ ratio of $\mathrm{T}-300$ fibers. The results of the material property predictions yielded the TSP presented in the following column. The final two columns are the calculated peak temperature results and the critical factor of safety in the spanwise direction for each architecture evaluated.

For all hybrid fiber architectures evaluated, the computed margins of safety were all greater than one, and thus all architectures are expected to survive the aerodynamic heating conditions. As compared to the baseline design, which was a 3:1 architecture of only K321 fibers with a predicted critical factor of safety of 0.42 , the advantage of hybrid architectures for leading edge design are evident.

The most significant increase in the factor of safety was observed for the architecture with the 1:0 layers, i.e., which contained no spanwise fibers. When spanwise fibers are then introduced into the architecture, the factor of safety dropped significantly. Further, compared to the same weave ratio with no 45-degree layers, the addition of 45-degree layers of T-300 fibers did not lead to any significantly improvement in the factor of safety. More significantly, adding 45-degree layers to the $3: 1$ and $4: 1$ hybrids resulted in an 
Table 1. Hybrid Fiber Architecture Evaluation Results

\begin{tabular}{|c|c|c|c|c|}
\hline \multicolumn{2}{|c|}{$\begin{array}{c}\text { HYBRID FIBER } \\
\text { ARCHITECTURE }\end{array}$} & $\begin{array}{c}\text { MATERIAL } \\
\text { ANALYSIS }\end{array}$ & \multicolumn{2}{|c|}{ FINITE ELEMENT ANALYSIS } \\
\cline { 4 - 5 } Weave & $\begin{array}{c}\% \pm 45^{\circ} \\
\mathrm{T}-300 \text { fibers }\end{array}$ & $\begin{array}{c}\mathrm{F}_{\mathrm{cy}} \mathrm{k}_{\mathrm{x}} \\
\mathrm{E}_{\mathrm{y}} \alpha_{\mathrm{y}}\end{array}\left(\frac{\mathrm{Btu}}{\mathrm{in}-\mathrm{sec}}\right)$ & $\begin{array}{c}\text { Thax } \\
\left({ }^{\circ} \mathrm{F}\right)\end{array}$ & FS $_{\text {critical }}$ \\
\hline \multirow{2}{*}{$1: 0$} & 33.3 & 5.19 & & \\
\cline { 2 - 5 } & 50 & 6.39 & 3800 & 2.12 \\
\hline $1: 1$ & 0 & 4.83 & 4050 & 1.16 \\
\hline $2: 1$ & 0 & 4.04 & 3820 & 1.08 \\
\hline \multirow{2}{*}{$3: 1$} & 0 & 3.87 & 3720 & 1.09 \\
\cline { 2 - 5 } & 20 & 3.74 & 3810 & 1.08 \\
\hline \multirow{2}{*}{$4: 1$} & 0 & 3.62 & 3660 & 1.07 \\
\cline { 2 - 5 } & 28.6 & 3.59 & 3810 & \\
\hline $5: 1$ & 44.4 & 3.53 & 3900 & \\
\hline
\end{tabular}

increase in the predicted peak temperature, which is undesirable for the leading edge designs.

From all architectures evaluated, the predicted TSP was observed to be maximum for the $1: 0$ architecture containing $50 \%$ of $\pm 45^{\circ} \mathrm{T}-300$ fibers. The result of the maximum TSP correlated well with the maximum $\mathrm{FS}_{\text {critical }}$ predicted from the finite element analysis. Although the overall trend showed that TSP decreased with decreasing FS, there were some minor discrepancies observed, where the FS $_{\text {critical }}$ showed improvement as TSP decreased within a given weave. Concurrent with the observation of the slight increase in FS while TSP decreased was that $\mathrm{T}_{\max }$ increased. Consequently, the undesirable increase in peak temperature was also captured with the TSP decreasing slightly. The conclusion can then be inferred that material system ranking roughly correlates with the TSP.

\section{VALIDATION TESTING}

Experimental validation is required to both ensure the ability of the leading edge to operate without failure when subject to the aerodynamic flight loads and also to validate the methodology used to predict behavior.

\section{Material Property Tests}

Validation testing on the predicted material properties is required to refine the prediction technique for hybrid fiber architecture $\mathrm{C} / \mathrm{C}$ material systems and experimentally correlate the predicted TSP. Since the key material properties affecting the thermal structural response of the sharp leading edge are reasoned to be $\mathrm{k}_{\mathrm{x}}, \mathrm{E}_{\mathrm{y}}$, $F_{y}$, and $\alpha_{y}$, preliminary material property testing should be limited to these properties to efficiently screen initial materials under consideration. Having experimental data for the key properties can then be further utilized to predict to greater accuracy the other properties necessary for the three-dimensional analysis of the leading edge.

To verify material properties, fabrication of a ten inch by ten inch panel that is 0.21 inches thick, as illustrated in Figure 6, should be sufficient. On the panel the specimens shaded in gray are those needed for the initial key property measurements. The test matrix corresponding to the key properties is presented in Table 2. The compression test will provide the $\mathrm{E}_{\mathrm{y}}$ and $\mathrm{F}_{\mathrm{y}}$ properties with four replicates at the two temperatures of $70^{\circ} \mathrm{F}$ and $3800^{\circ} \mathrm{F}$. The expansion test will provide $\alpha_{\mathrm{y}}$ between $70^{\circ} \mathrm{F}$ and $3800^{\circ} \mathrm{F}$ through two replicate tests. The thermal conductivity test will provide the instantaneous $\mathrm{k}_{\mathrm{x}}$ at several temperatures between $70^{\circ} \mathrm{F}$ and $3000^{\circ} \mathrm{F}$, which should be sufficient for this property where material property predictions should suffice for higher temperature variations. 


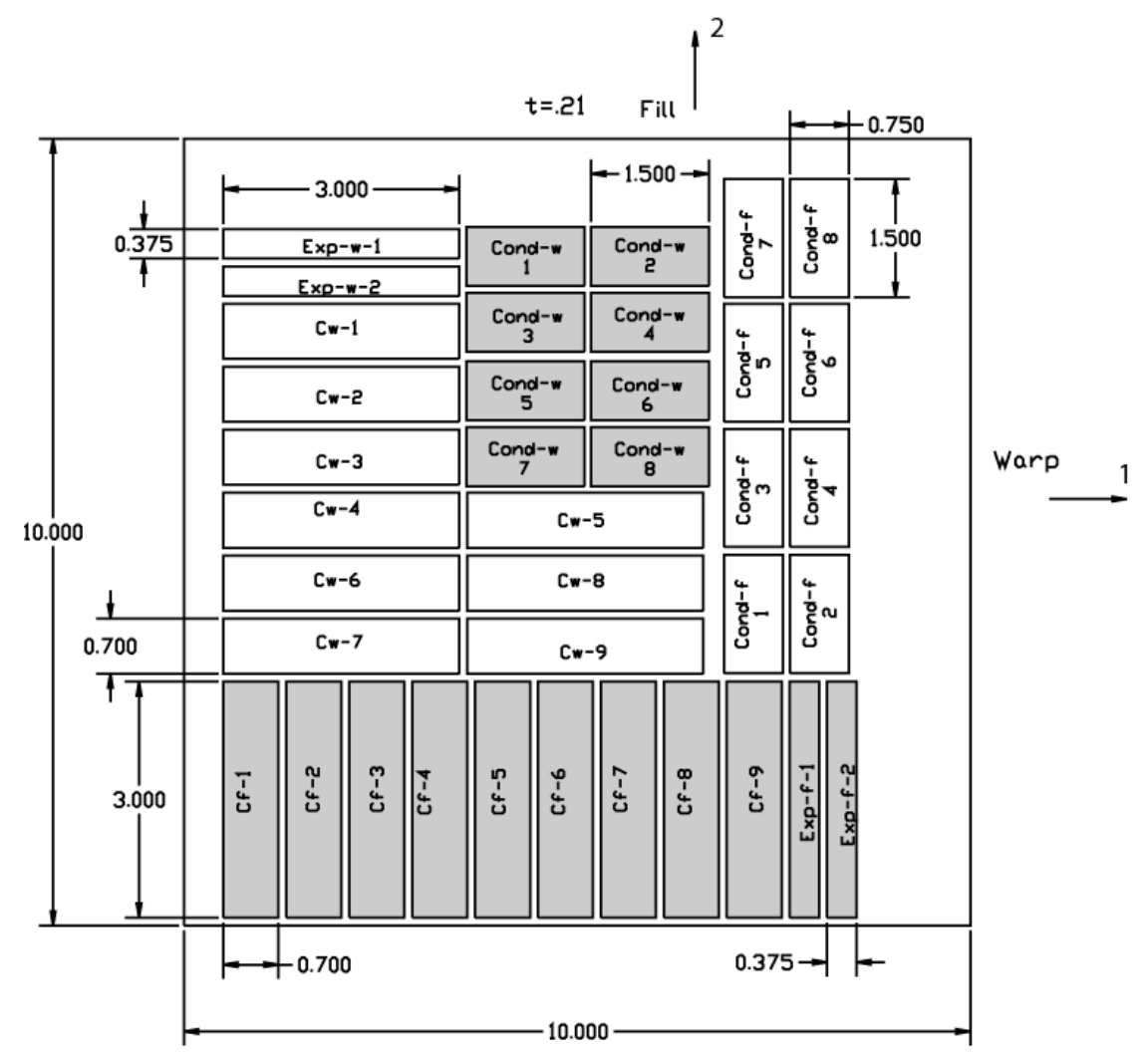

Figure 6. Material property test panel (all dimensions are in inches).

Table 2. Test Matrix to be repeated for each panel

\begin{tabular}{|l|c|c|c|c|}
\hline \multicolumn{1}{|c|}{ Description } & $70^{\circ} \mathrm{F}$ & $3000^{\circ} \mathrm{F}$ & $3800^{\circ} \mathrm{F}$ & Total \\
\hline Compression-Fill (Cf) & 4 & & 4 & 8 \\
\hline Thermal Expansion - Fill (Exp-f) & 2 & & & 2 \\
\hline Thermal Conductivity- Warp (Cond-w) & 2 & $>$ & & 2 \\
\hline & & & & 12 \\
\hline
\end{tabular}

\section{Leading Edge Test Specimens}

For consistency with the properties, leading edge specimens are also being fabricated by the same vendor as the material property panels utilizing the same batch of fibers. The pertinent issues regarding the test specimen include what a minimally acceptable spanwise width should be for replication of the critical state of thermal stress. The issue arose from the ability of test facilities to accommodate large specimens in addition to the increased costs associated with larger specimens. Addressing this issue, finite element analyses were conducted on varying specimen width. The results of varying specimen width on the critical thermal stress are presented in Figure 7. From Figure 7, the observation is made that once a specimen width of about 8 inches is reached, the peak stress levels off without any further increase with increasing width. Therefore, for the current design, a specimen width of 8 inches will be required to reach the peak design thermal stresses for evaluation. 

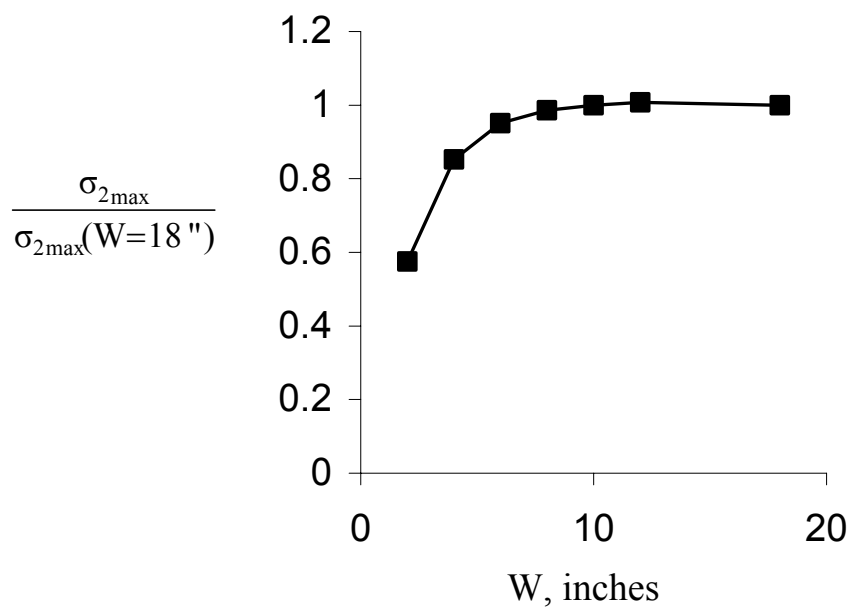

Figure 7. Effect of specimen width on peak critical stress.

\section{FABRICATION OF TEST PANELS}

Currently, test panels for both material property and leading edge specimens are being fabricated under contract. Material specifications include:

1) Required heat treatment $=2700^{\circ} \mathrm{C}$

2) Minimum composite density $=0.065 \mathrm{lb} / \mathrm{in}^{3}$

3) Fiber volume fraction $=0.55$

To insure highest possible thermal conductivity, the $\mathrm{C} / \mathrm{C}$ composites are to be fabricated using a combination of pitch matrix densification and chemical vapor infiltration.

The two fiber architectures chosen for fabrication include the 1:0 architecture with 50\% $45^{\circ} \mathrm{T}-300$ fibers and the 3:1 hybrid architecture with zero $45^{\circ}$ plies. The material property test panel fabrication is complete. Preliminary inspection revealed the panels to be of quality construction and material property testing is underway.

\section{CONCLUDING REMARKS}

This paper presented work underway for the development of sharp refractory composite leading edges for advanced hypersonic vehicles. A vehicle nose leading edge for a specific Mach
10 flight vehicle trajectory was used as a basis for this study. Detailed thermal-structural finite element analyses for a baseline material revealed that the use of a $\mathrm{C} / \mathrm{C}$ material system with a single fiber type would not survive the application. Based on the predicted thermal-structural response, the use of a hybrid fiber architecture was identified to be worthy of investigation. Material property predictions were subsequently conducted for several hybrid architectures. A tailored thermal shock parameter for sharp orthotropic leading edges was derived in an attempt to identify a material parameter to preliminarily screen fiber architectures. Although the parameter shows promise in identifying the optimal material systems, further investigation is underway to experimentally verify the predicted parameter and further evaluate the assumptions used with the computation.

Key material property tests and the requirements for test panels necessary to evaluate the advanced hybrid fiber architectures were defined. The leading edge test specimen width required to produce the critical thermal stress magnitude was identified. Material property test panels have been successfully fabricated with hybrid architectures and validation testing is currently in progress. 


\section{REFERENCES}

1. Glass, D. E., Merski, N. R., Glass, C.E., Airframe Research and Technology for Hypersonic Airbreathing Vehicles, NASA TM20020211752 , July 2002.

2. MSC/PATRAN/NASTRAN, Version 7, Vol. 1, The MacNeal-Schwendler Corperation, Los Angeles, CA, July, 1997.

3. B.J. Sullivan and K.W. Buesking, Data Correlation and Evaluation of Affordable CarbonCarbon Composites:Effective Properties of K321 and P-30x Fibers in C-C Composites, Proceedings of the $21^{\text {st }}$ Annual Conference on Composites, Materials and Structures, Cocoa Beach, FL, January 1997.

4. J. Spain and S. Starrett, Mechanical and Thermal Evaluation of Coated Carbon-Carbon, Southern Research Institute Final Report to General Dynamics Fort Worth Division, SRI-ME91-271-6913, April 1991.

5. Z. Hashin, Analysis of Properties of Fiber Composites with Anisotropic Constituents, J. Appl. Mech., Vol. 46, 1979, pp. 543-550.

6. N.J. Pagano and G.P. Tandon, Elastic Response of Multidirectional Coated Fiber Composites, Composites Science and Technology, Vol. 31, 1988, 273-293.

7. B.W. Rosen, et al., An Analysis Model for Spatially Oriented Fiber Composites, Composite Materials Testing and Design, ASTM STP 617, 1977, pp. 243-259.

8. Lidman, W.G. and Bobrowsky, A. R., Correlation of Physical Properties of Ceramic Materials with Resistance to Fracture by Thermal Shock, NACA TN 1918, July 1949.

9. Wang, Y. R. and Chou, T. W., Thermal Shock Resistance of Laminated Ceramic Matrix Composites, Journal of Material Science, 26, 1991, pp. 2961-2966.

American Institute of Aeronautics and Astronautics 